\title{
Tendon-to-bone healing using autologous bone marrow-derived mesenchymal stem cells in ACL reconstruction without a tibial bone tunnel -A histological study-
}

\author{
Tomonoshin Kanazawa ${ }^{1,2}$ \\ Takashi Soejima ${ }^{1}$ \\ Kouji Noguchi ${ }^{1}$ \\ KousukeTabuchi ${ }^{1}$ \\ Megumi Noyama ${ }^{1}$ \\ Kei-ichiro Nakamura² \\ Naoto Shiba ${ }^{1}$
}

\footnotetext{
1 Department of Orthopaedic Surgery, Kurume University School of Medicine, Fukuoka, Japan

2 Division of Microscopic and Development Anatomy, Department of Anatomy, Kurume University School of Medicine, Fukuoka, Japan
}

Corresponding author:

Tomonoshin Kanazawa

Division of Microscopic and Development Anatomy, Department of Anatomy

Kurume University School of Medicine

67 Asahi-machi, Kurume City, Fukuoka, Japan

E-mail: tomochan@med.kurume-u.ac.jp

\section{Summary}

Background: after anterior cruciate ligament $(A C L)$ reconstruction, it is necessary to integrate free tendon graft biologically to the bone. In the present study, to verify whether a structure identical to the normal ligament-bone insertion could be regenerated at the tendon-bone interface without bone tunnel, we designed $A C L$ reconstruction model without a tibial bone tunnel. Moreover, to enhance the integration process in this model, bone marrow-derived mesenchymal stem cells (bMSCs) were transplanted, and histological changes investigated.

Our first hypothesis was that the grafted tendon would be anchored at part of the tendon-bone interface even if a bone tunnel was not created. Second hypothesis was that application of bMSCs at the tendon-bone interface would yield results histologically superior to those in controls.

Methods: bilateral ACL reconstruction using our originally designed method was performed. Autologous bMSCs with the carrier were transplanted between the bottom of the grafted tendon and the bone pit of the tibia in the experimental limb, whereas the control limb received the carrier on- ly. At 4 and 8 weeks after the operation, histological comparison between bMSCs and the control group was carried out.

Results/Conclusions: even in our present ACL reconstruction model without a tibial bone tunnel, integration via chondroid tissue was seen at part of the tendon-bone interface. However, there were no appreciable differences between the groups. In ACL reconstruction, to enhance the tendonbone integration without a bone tunnel would lead to save the graft length and prevent from bone tunnel complications (ex. Bone-tunnel enlargement after surgery).

KEY WORDS: anterior cruciate ligament, enthesis, mesenchymal stem cell, rabbits, tendon-to-bone healing, tunnel enlargement.

\section{Introduction}

To obtain a successful outcome after anterior cruciate ligament $(A C L)$ reconstruction, a free tendon graft requires to be anchored securely and biologically to the bone. However, the histological structure at the tendon-bone interface is totally different from the highly specialized normal ligament-bone insertion ${ }^{1,2}$.

On the other hands, tunnel enlargement within the bone tunnel has been reported after ACL reconstruction, and it causes various clinical complications ${ }^{3,4}$. Tunnel enlargement within the bone tunnel obviously has the risk of the graft failure, furthermore, the bone loss could be also the interruption of the revision surgery. Previous studies have been shown that the grafted tendon is initially integrated via fibrous tissue within the bone tunnel wall to resist shear stress. This fibrous interface tissue contains characteristic anchoring fibres known as Sharpey-like fibres which have been shown to be correlated with pull-out strength ${ }^{5,6}$. The amount of these fibres gradually increases, and their major constituent changes from type III to type I collagen 7 . However, they are not uniformly present at all sites in the bone tunnel until the final stage ${ }^{8}$. Thereafter, integration at the entrance of the bone tunnel occurs partly at compressive sites via chondroid tissue, but mostly occurs via fibrous tissue $^{9,10}$. Once the graft is immobilized at the entrance, myxoid degeneration occurs within the bone tunnel graft, due to stress shielding 7,10 .

These phenomena have two possible interpretations. Although temporary anchoring within the bone tunnel 
would be necessary initially, it would no longer be required once integration at the entrance has been established. If compressive force is applied at the entrance, chondroid formation would be promoted. Therefore, we hypothesize that the grafted tendon would become anchored via chondroid tissue when the graft was press-fitted to the bone surface, even if a bone tunnel was not created. Additionally, the tendon-bone integration without the bone tunnel would lead to save the graft length and prevent from these bone tunnel complications.

In the present study, therefore, to verify whether a structure identical to the normal ligament-bone insertion could be regenerated at the tendon-bone interface without a bone tunnel, we designed an original ACL reconstruction model without a tibial bone tunnel and investigated the histological changes between the bottom of the grafted tendon and the tibial bone. However, it is thought that the biomechanical strength of the tendon bone interface is obviously weaker than the conventional bone tunnel model, and then some kind of biological approach is necessary in addition to a suitable structural or mechanical environment, to re-create the histological structure near the native insertion site without the formation of fibrous tissue. Previous studies have shown that bone marrow-derived mesenchymal stem cells (bMSCs) have promising ability to augment tendon graft healing in a bone tunnel ${ }^{11,12}$. bMSCs are multipotent cells that can differentiate into multiple mesenchymal tissues ${ }^{13}$. Therefore, to enhance the integration process in our model, we transplanted bMSCs between the bottom of the grafted tendon and the tibial bone.

Our first hypothesis was that the grafted tendon would be anchored via chondroid tissue at part of the tendon-bone interface even if a bone tunnel was not created. Our second hypothesis was that application of bMSCs at the tendon-bone interface would yield results histologically superior to those in controls without bMSCs.

\section{Material and methods}

\section{Experimental design}

14 skeletally mature Japanese white rabbits (weighing 2.5 to $3.0 \mathrm{~kg}$ ) were used. All animals were ethically conducted according to the international standards ${ }^{14}$. Ethical approval was obtained from the Animal Care Center of Kurume University. Autologous bMSCs were harvested from the bone marrow of the rabbits 2 weeks before surgery. For bilateral ACL reconstruction using our originally designed method described later, bMSCs in collagen sponge or a fibrin sealant carrier were transplanted between the bottom of the grafted tendon and the bone pit of the tibia in the experimental limb, whereas the control limb received the carrier only. The animals were euthanatized at 4 and 8 weeks after the operation, and 7 rabbits at each time point were used for histological analysis.

\section{MSC preparation}

The rabbits were anesthetized, and 5 to $10 \mathrm{ml}$ of bone marrow was aspirated from the posterior iliac crest under sterile conditions. The anti-coagulated bone marrow was diluted with twice the volume of Hank's Balanced Salt Solution. The nucleated cell layer was obtained by Ficoll-Paque density gradient centrifugation (Sigma Chemical, St. Louis, MO) at $500 \times \mathrm{g}$ for $20 \mathrm{~min}$. The nucleated cells were resuspended in STK-1 (DS Pharma Biomedical, Osaka, Japan) containing penicillin $100 \mathrm{U} / \mathrm{mL}$ and, streptomycin $100 \mathrm{U} / \mathrm{mL}$. The number of nucleated cells was determined using a hemocytometer, and the volume of the suspension was adjusted. The cells were plated out at a density of $2.0 \times 10^{5} / \mathrm{cm}^{2}$ in a 6 well plate and grown at $37^{\circ} \mathrm{C}$, under a $5 \% \mathrm{CO} 2$ in a humidified tissue-culture incubator. The medium was changed every 2 days. After 10 days, the cells were detached and transferred to 6-well plates with STK-2 (DS Pharma Biomedical, Osaka, Japan). About 10 million cells were obtained for use in each model.

\section{Surgical protocol}

Our originally designed ACL reconstruction procedure was performed bilaterally under general anesthesia. A midline skin incision was made and subcutaneous tissues were divided. The semitendinosus tendon was harvested and folded in half. A suture was passed through free end of the graft using Krackow fashion. For the loop end of the graft, a suture was passed through the graft $2 \mathrm{~mm}$ from the loop end and turned twice around the graft, then securely tied with a double surgeon's knot. Then, one end of the suture was passed again through the graft to the reverse side of the knot. A medial parapatellar arthrotomy was used to expose the knee joint, and the native $A C L$ was excised. Using a $4-\mathrm{mm}$ bit grinder, a bone pit ( $4 \mathrm{~mm}$ in diameter, $2 \mathrm{~mm}$ in depth) was created at the tibial footprint of the ACL, and a small hole (less than $2 \mathrm{~mm}$ in diameter) for pull-out was created in the center of the bone pit. At the femoral footprint, a bone tunnel was created using 4-mm-bit drill. Once the graft had been drawn into the femoral bone tunnel from the Krackow stitch side, the suture near the loop end of the graft was pulled out to the tibial hole and firmly secured to a metal button on the anterior cortex of the tibia so that a 2-mm margin of the loop end would be retained in the bone pit under a suitable pressure. At this point, collagen sponge or fibrin sealant containing bMSCs was transplanted between the tibial bone pit and the grafted tendon in the experimental group (Fig. 1). The control group received a transplant of the carrier only. Finally, a suture at the free end of the graft was secured to a metal button on the femoral cortex under slight tension. The wound was then closed in layers. The animals were allowed to move freely in their cages after the operation. 


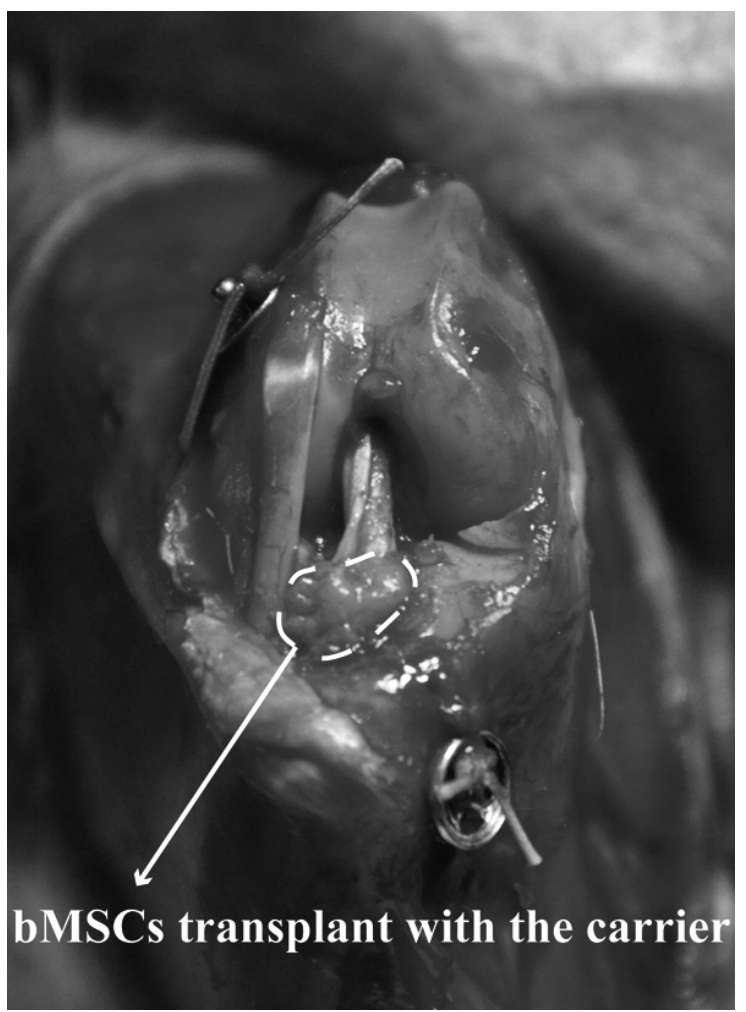

Figure 1. ACL reconstruction without a tibial bone tunnel. After the graft had been passed through the bone tunnel in the femur, the carrier containing bMSCs was transplanted between the tibial bone pit and the grafted tendon under tensioning (white arrow).

\section{Histologic analysis}

After removal of the graft-tibia complex, the specimens were fixed in $10 \%$ buffered formalin. Decalcification was performed using $30 \%$ formic acid. The samples were embedded in paraffin and cut into $5-\mu \mathrm{m}$ thick sections longitudinal to the grafted tendon and the tibial bone. The slides were stained using hematoxylin and eosin and, safranin-O, and also stained immunohistochemically for collagen type I, II, and III.

\section{Immunohistological protocol}

Immunostaining was performed with monoclonal antibodies against collagen type I, II, and III (Daiichi, Toyama, Japan) according to the Envision method. After deparaffinization of the tissue sections, activation of antigens was conducted using $3 \%$ bovine serum albumin for $30 \mathrm{~min}$ at room temperature. Blocking of endogenous peroxidase was then conducted by incubation with methanol containing $3 \% \mathrm{H} 2 \mathrm{O} 2$ for 30 min at room temperature. The primary antibody was diluted to an appropriate concentration with Anti-Diluent Buffer (DAKO, S3022), and each reaction was performed overnight at $4^{\circ} \mathrm{C}$. As a secondary antibody for the Envision method, an anti-mouse enzyme marker dextran polymer (DAKO, K4000) for the monoclonal antibody was allowed to react for $30 \mathrm{~min}$ at room temperature. As a chromogenic substrate, DAB (3,3'-diaminobenzidine tetrahydrochloride) was used for coloration. After antibody staining, the sections were stained with hematoxylin, dehydrated, and cleared. In each process, the cleaning solution used was $0.1 \%$ Tween 20 and, $0.3 \mathrm{~mol} / \mathrm{L} \mathrm{NaCl}$ in $0.05 \mathrm{~mol} / \mathrm{L}$ Tris- $\mathrm{HCl}$ buffer. The resulting sections were observed using an optical microscope. Normal mouse IgG1 (DAKO, X0931), adjusted to the concentration of the primary antibody, was used as a negative control.

\section{Histological evaluation}

The tendon-bone interface in each specimen was divided into anterior and posterior parts, and histological comparison between bMSCs and the control group was carried out. Differences in the frequencies of chondroid cell layer formation in each group were investigated.

\section{Results}

\section{Histology}

At 4 weeks, in the control group, poorly organized fibrovascular tissue consisting of type III collagen was evident between the grafted tendon and the bone (Fig. 2 a,c). On the other hand, in the bMSCs group, active chondroid cells had appeared from the wall of the bone pit, and showed gradual maturation into bone in a few cases (Fig. 2 b,d).

At 8 weeks, in the control group, the interface was less cellular and had become progressively organized in comparison with the situation at 4 weeks (Fig. 3 $a, b, c)$. However, few active chondroid layers consisting of type II collagen and staining reddish purple with safranin-O was seen. In contrast, in the bMSCs group, partial integration was seen between the grafted tendon and the bone, and this integration was more evident at the posterior than at the anterior interface. Moreover, an active chondroid cell layer was observed throughout the area between the grafted tendon and the bone in one case (Fig. $3 \mathrm{~d}, \mathrm{e}, \mathrm{f}$ ).

\section{Chondroid cell layer formation}

At 4 weeks, in the bMSCs group, a chondroid cell layer stained with safranin-O and showing immunoreactivity for type II collagen was observed at the posterior interface in two of seven specimens, compared with only one of seven specimens from the control side. No chondroid cell layer was observed at the anterior interface in either group. In one of seven control specimens, a chondroid cell layer was observed at the posterior interface. No chondroid cell layer was observed at the anterior interface. 

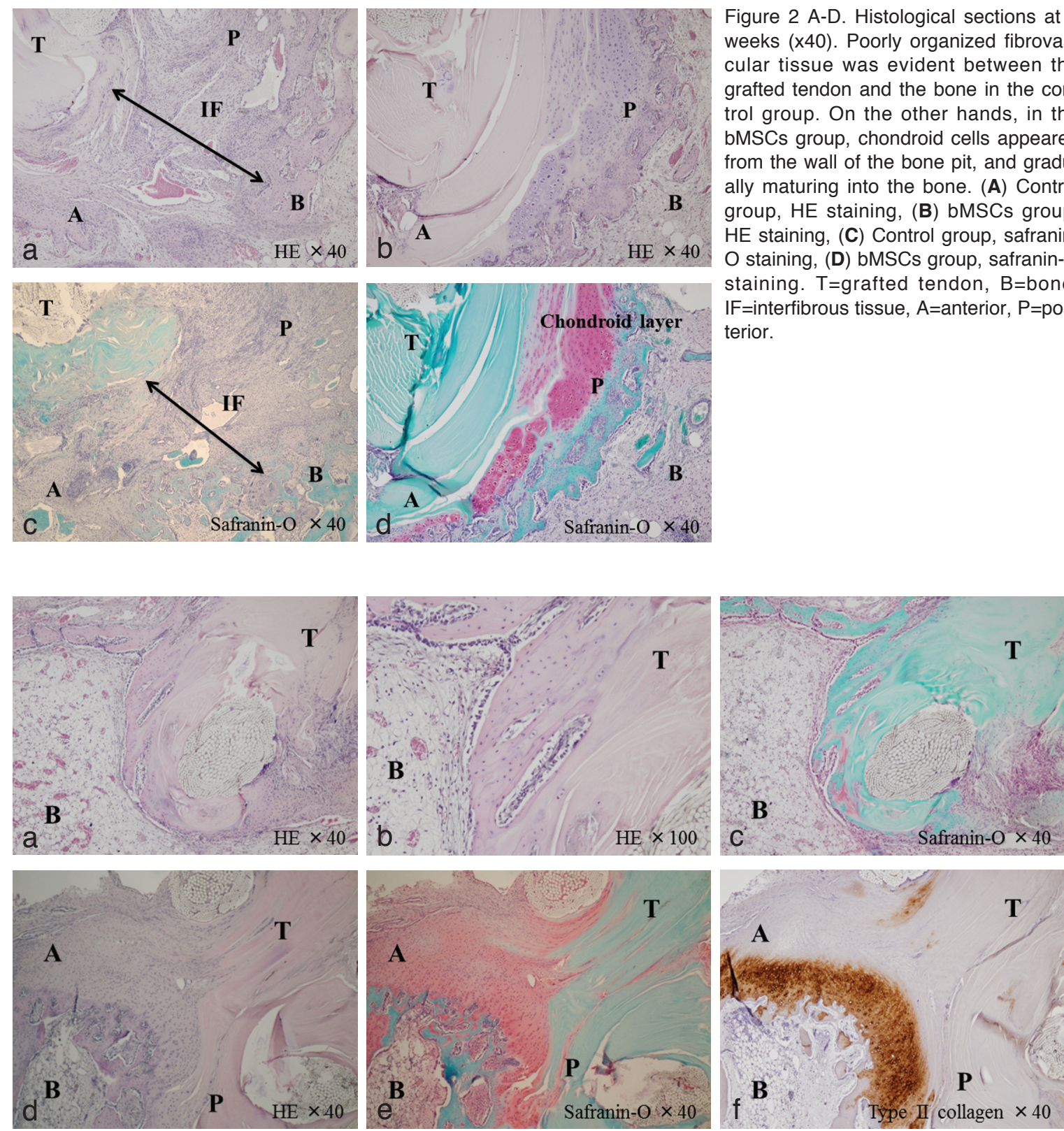

Figure 2 A-D. Histological sections at 4 weeks (x40). Poorly organized fibrovascular tissue was evident between the grafted tendon and the bone in the control group. On the other hands, in the bMSCs group, chondroid cells appeared from the wall of the bone pit, and gradually maturing into the bone. (A) Control group, HE staining, (B) bMSCs group, HE staining, (C) Control group, safraninO staining, (D) bMSCs group, safranin-O staining. $T=$ grafted tendon, $B=b o n e$, $\mathrm{IF}=$ interfibrous tissue, $\mathrm{A}=$ anterior, $\mathrm{P}=$ posterior.

Figure 3 A-F. Histological sections at 8 weeks. The tendon-bone interface was less cellular and had become progressively organized in comparison with the situation at 4 weeks. (A) Control group, HE staining (x40), (B) Control group, HE staining (x100), (C) Control group, safranin-O staining (x40), (D) bMSCs group, HE staining (x40), (E) bMSCs group, safranin-O staining (x40), (F) bMSCs group, type II collagen immunostaining (x40).

At 8 weeks, in the bMSCs group, a chondroid cell layer was more obvious at the posterior interface in five of seven specimens, compared with the situation at 4 weeks. In addition, a chondroid cell layer was evident at both the anterior and posterior interfaces in only one specimen. Two of seven control specimens showed a chondroid cell layer at the posterior interface, but no such layer was evident at the anterior interface in any of the specimens. A summary of the histological analysis of chondroid cell layer formation is shown in Table 1.

\section{Discussion}

Even in our present ACL reconstruction model without a tibial bone tunnel, integration via chondroid tissue was seen at part of the tendon-bone interface, and furthermore, the integration was more localized at the posterior than at the anterior interface. Our first hypothesis that the bone tunnel was not an essential factor for tendon-to-bone integration was thus verified. For integration of a grafted tendon with bone, the mechanical environment at the tendon-bone interface is 
Table 1. A summary of the histological analysis of chondroid cell layer formation.

\begin{tabular}{|l|c|c|c|c|}
\hline \multirow{2}{*}{} & \multicolumn{2}{|c|}{ PO4W $(\mathrm{n}=7)$} & \multicolumn{2}{c|}{ PO8W $(\mathrm{n}=7)$} \\
\cline { 2 - 5 } & Anterior & Posterior & Anterior & Posterior \\
\hline bMSCs group & $0 / 7$ & $2 / 7$ & $1 / 7$ & $5 / 7$ \\
\hline Control group & $0 / 7$ & $1 / 7$ & $0 / 7$ & $2 / 7$ \\
\hline
\end{tabular}

one of the major contributory factors. Previous studies have also shown that the contact area between soft tissue and bone is a major determinant of healing ${ }^{15-17}$. In the present study, the contact area between the grafted tendon and the bone was smaller than that in a conventional bone tunnel model. Therefore, the initial mechanical strength in the absence of a bone tunnel would obviously have been weaker than that in the conventional bone tunnel model. However, in an analysis of the relationship between mechanical stress and tendon-to-bone healing, Yamakado et al. noted that tensile stress enhances tendon-bone healing, compressive stress promotes chondroid formation, and shear load has little or no effect on regeneration of the tendon-bone junction ${ }^{10}$. In the present study, we did not create a tibial bone tunnel, and retained the graft end in bone pit under a suitable pressure. Therefore it was expected that the grafted tendon-bone interface would be exposed to compressive force in the static phase and to tensile force in the dynamic phase. On the other hand, in the conventional bone tunnel model, it is thought that the bone tunnel would be exposed to shear stress. Additionally, the histological changes including the types of collagen revealed by immunostaining were almost the same as those observed in previous reports ${ }^{18}$. These factors indicate that if rigid fixation to prevent failure of the grafted tendon is achieved at an early stage, then the mechanical environment created in the absence of a bone tunnel might be superior to that in the conventional bone tunnel model.

It is known that the initial histological change on the tendon-to-bone healing via Sharpey-like fibres was approximately converged until 8 weeks 6,18 . In this study, the initial histological change after bMSCs transplant needed to be investigated, therefore time points of the evaluation were set out at 4 and 8 weeks.

There are several possible explanations for why the integration was more localized at the posterior interface than at the anterior interface. Other studies have noted that compressive stress promotes chondroid formation ${ }^{9}, 10$. In a natural state, rabbits hold the knee highly flexed, and therefore, the posterior interface is thought to be more exposed to compressive stress, thus promoting chondroid layer formation.

On the other hand, there were no appreciable differences between the groups. Initially, we hypothesized that application of bMSCs would increase the amount of fibrocartilage formation, or provide fibrous tissue anchorage between the bottom of the grafted tendon and the bone, and furthermore, might promote regeneration like the native ligament-bone structure. How- ever, our second hypothesis was disproved, at least under the specific conditions used for this study. Although many previous researchers have verified tendon-to-bone integration utilizing MSCs ${ }^{11}, 12,19,20$ the properties of MSCs in vivo still remain unknown. Lim et al. reported that coating of tendon grafts with MSCs resulted in healing through formation of an intervening chondroid layer resembling the chondralenthesis of normal ACL insertions, rather than collagen fibers and scar tissue, yielding a performance significantly better than controls in biomechanical testing ${ }^{11}$. In contrast, Gulotta et al. reported that the addition of MSCs alone to the healing rotator cuff insertion site did not improve the structure, composition, or strength of the healing tendon attachment site, and speculated that cell-based strategies may require the use of appropriate growth and differentiation factors ${ }^{19}$. In the present study, we performed autologous transplantation unlike some previous studies ${ }^{19,21}$, and used about 10 million cells, which was a greater number than those used in previous studies ${ }^{11,12}$. As autologous transplantation is obviously superior to allogenic transplantation, and a sufficient number of bMSCs were transplanted, we believe that the quantity and quality of the MSCs used in our study were more appropriate than in previous studies 11, 12, 19, 21. Nevertheless, an active chondroid cell layer between the grafted tendon and the bone was not observed, except in only one case. We might have failed to control the differentiation of the transplanted cells and capacitate them sufficiently in vivo. However, the potential utility of bMSCs could not be ruled out, since at least only one of it was proved. In order to accelerate tendon-to-bone integration or to regenerate the histological structure at the ligament-bone insertion utilizing MSCs, further studies will be required to determine not only the applicability of MSCs alone, but also whether their proliferation/differentiation can be controlled with either growth or differentiation factors. In addition, many previous researchers have attemped to enhance/accelerate tendon-to-bone healing using various materials 22,23 , but few have analyzed the structure and development at the normal insertion $^{1,2,24,25}$. Consequently, details of the normal ultrastructure at the ligament/tendon-bone insertion still remain unclear. To regenerate the normal insertion, it will also be necessary to clarify in more detail the normal ligament/tendon-to-bone structure and its biological development.

There were several limitations to this study. First, the findings obtained using a rabbit model would differ appreciably from the situation in humans. Second, we examined the treated animals only up to 8 weeks because the grafted tendon is known to fail at the tendonbone junction at an early time point, particularly within 8 weeks ${ }^{22}$. However, if we had extended our observation period, some beneficial effects on tendon-to-bone healing might have become evident. Finally, the results obtained were mainly qualitative, and no biomechanical tests or quantitative evaluations were performed. In conclusion, in this ACL reconstruction model without the use of a bone tunnel, integration via chon- 
droid tissue was seen in part of the tendon-bone interface, indicating that the bone tunnel was not an essential factor for tendon-to-bone integration. However, the application of bMSCs alone was unable to improve tendon-to-bone healing to a sufficient extent. Further studies will be required to determine how best to control the differentiation of bMSCs, and to clarify details of the structure and biological development of the normal ligament/tendon-bone interface to facilitate its effective regeneration.

\section{Acknowledgement}

The authors thank Mr. Kazuhiro Yoshida for his technical assistance. This work was supported by a Grant-in Aid for Scientific Research C: KAKENHI 20591769.

\section{References}

1. Benjamin M, Evans EJ, Copp L. The histology of tendon attachments to bone in man. J Anat. 1986;149:89-100.

2. Cooper RR, Misol S. Tendon and ligament insertion. A light and electron microscopic study. J Bone Joint Surg Am. 1970;52:1-20

3. Quatman CE, Paterno MV, Wordeman SC, Kaeding CC. Longitudinal anterior knee laxity related to substantial tibial tunnel enlargement after anterior cruciate ligament revision. Arthroscopy. 2011;27:1160-1163.

4. Wilson TC, Kantaras A, Atay A, Johnson DL. Tunnel enlargement after anterior cruciate ligament surgery. Am J Sports Med. 2004;32:543-549.

5. Goradia VK, Rochat MC, Grana WA, Rohrer MD, Prasad HS. Tendon-to-bone healing of a semitendinosus tendon autograft used for ACL reconstruction in a sheep model. The American journal of knee surgery. 2000;13:143-151.

6. Rodeo SA, Arnoczky SP, Torzilli PA, Hidaka C, Warren RF. Tendon-healing in a bone tunnel. A biomechanical and histological study in the dog. J Bone Joint Surg Am. 1993;75:17951803.

7. Tabuchi K, Soejima T, Kanazawa T, Noguchi K, Nagata K. Chronological changes in the collagen-type composition at tendon-bone interface in rabbits. Bone Joint Res. 2012;1:218224.

8. Tomita F, Yasuda K, Mikami S, Sakai T, Yamazaki S, Tohya$\mathrm{ma} \mathrm{H}$. Comparisons of intraosseous graft healing between the doubled flexor tendon graft and the bone-patellar tendon-bone graft in anterior cruciate ligament reconstruction. Arthroscopy. 2001;17:461-476.

9. Mutsuzaki H, Sakane M, Nakajima H, Ochiai N. Calcium phosphate-hybridised tendon graft to reduce bone-tunnel enlargement after ACL reconstruction in goats. Knee. 2011;19:455460.

10. Yamakado K, Kitaoka K, Yamada H, Hashiba K, Nakamura R, Tomita K. The influence of mechanical stress on graft healing in a bone tunnel. Arthroscopy. 2002;18:82-90.

11. Lim JK, Hui J, Li L, Thambyah A, Goh J, Lee EH. Enhance- ment of tendon graft osteointegration using mesenchymal stem cells in a rabbit model of anterior cruciate ligament reconstruction. Arthroscopy. 2004;20:899-910.

12. Ouyang HW, Goh JC, Lee EH. Use of bone marrow stromal cells for tendon graft-to-bone healing: histological and immunohistochemical studies in a rabbit model. Am J Sports Med. 2004;32:321-327.

13. Chamberlain G, Fox J, Ashton B, Middleton J. Concise review: mesenchymal stem cells: their phenotype, differentiation capacity, immunological features, and potential for homing. Stem cells (Dayton, Ohio). 2007;25:2739-2749.

14. Padulo J, Oliva F, Frizziero A, Maffulli N. Muscles, Ligaments and Tendons Journal. Basic principles and recommendations in clinical and field science research. MLTJ. 2013;4:250-252.

15. Greis PE, Burks RT, Bachus K, Luker MG. The influence of tendon length and fit on the strength of a tendon-bone tunnel complex. A biomechanical and histologic study in the dog. Am J Sports Med. 2001;29:493-497.

16. Yamazaki S. The effect of Graft-Tunnel Diameter Disparity on Intraosseous Healing of the Flexor Tendon Graft in Anterior Cruciate Ligament Reconstruction. The American Journal of Sports Medicine. 2002;30:498-505.

17. Yamazaki S, Yasuda K, Tomita F, Minami A, Tohyama H. The effect of intraosseous graft length on tendon-bone healing in anterior cruciate ligament reconstruction using flexor tendon. Knee Surg Sports Traumatol Arthrosc. 2006;14:1086-1093.

18. Kanazawa T, Soejima T, Murakami H, Inoue T, Katouda M, Nagata K. An immunohistological study of the integration at the bone-tendon interface after reconstruction of the anterior cruciate ligament in rabbits. J Bone Joint Surg Br. 2006;88: 682-687.

19. Gulotta LV, Kovacevic D, Ehteshami JR, Dagher E, Packer JD, Rodeo SA. Application of bone marrow-derived mesenchymal stem cells in a rotator cuff repair model. Am J Sports Med. 2009;37:2126-2133.

20. Li YG, Wei JN, Lu J, Wu XT, Teng GJ. Labeling and tracing of bone marrow mesenchymal stem cells for tendon-to-bone tunnel healing. Knee Surg Sports Traumatol Arthrosc. 2011;19: 2153-2158.

21. Gulotta LV, Kovacevic D, Packer JD, Deng XH, Rodeo SA Bone marrow-derived mesenchymal stem cells transduced with scleraxis improve rotator cuff healing in a rat model. Am J Sports Med. 2011;39:1282-1289.

22. Mutsuzaki H, Sakane M, Hattori S, Kobayashi H, Ochiai N Firm anchoring between a calcium phosphate-hybridized tendon and bone for anterior cruciate ligament reconstruction in a goat model. Biomedical materials (Bristol, England). 2009;4: 045013.

23. Thomopoulos S, Matsuzaki H, Zaegel M, Gelberman RH, Silva MJ. Alendronate prevents bone loss and improves tendonto-bone repair strength in a canine model. J Orthop Res. 2007;25:473-479.

24. Galatz L, Rothermich S, VanderPloeg K, Petersen B, Sandell $\mathrm{L}$, Thomopoulos S. Development of the supraspinatus tendonto-bone insertion: localized expression of extracellular matrix and growth factor genes. J Orthop Res. 2007;25:1621-1628.

25. Thomopoulos S, Genin GM, Galatz LM. The development and morphogenesis of the tendon-to-bone insertion - what development can teach us about healing. Journal of musculoskeletal \& neuronal interactions. 2010;10:35-45. 